\title{
Mathematical Modeling and Kinematics Analysis of Double Spherical Shell Rotary Docking Skirt
}

\author{
Haixia Gong ${ }^{1}$, Zhe Liu ${ }^{1, a}$ and Liquan Wang ${ }^{1}$ \\ ${ }^{1}$ School of Mechanical and Electrical, Harbin Engineering University, 150001, China
}

\begin{abstract}
In order to solve the problem of large trim and heel angles of the wrecked submarine, the double spherical shell rotating docking skirt is studied. According to the working principle of the rotating docking skirt, and the fixed skirt, the directional skirt, the angle skirt are simplified as the connecting rod. Therefore, the posture equation and kinematics model of the docking skirt are deduced, and according to the kinematics model, the angle of rotation of the directional skirt and the angle skirt is obtained when the wrecked submarine is in different trim and heel angles. Through the directional skirt and angle skirt with the matching rotation can make docking skirt interface in the $0^{\circ} \sim 2 \gamma$ range within the rotation, to complete the docking skirt and the wrecked submarine docking. The MATLAB software is used to visualize the rotation angle of fixed skirt and directional skirt, which lays a good foundation for the development of the control of the double spherical shell rotating docking skirt in future.
\end{abstract}

\section{Introduction}

At present, underwater space station docking, marine life-saving are widely used underwater docking technology [1]. Underwater rescue mainly relies on life-saving vehicle equipped with docking skirt active docking wrecked submarine. Docking skirt becomes bridge of connection life rescue vehicle and the wrecked submarine. Most of the initial life-saving vehicle take a fixed docking skirt [2-3]. In recent years, foreign countries has developed rotating docking skirt [4]. Based on the working principle of rotating docking skirt. In this paper, a mathematical model is established and a kinematic model is derived, and finally verifies the correctness of the theoretical analysis by MATLAB.

\section{The working principle of rotating docking skirt}

Double spherical shell rotating skirt consists of three parts: fixed skirt, directional skirt and angle skirt [5], as is shown in Figure 1. Fixed skirt and life-saving vehicle is fixed connection, play the role of connecting directional skirt and life-saving vehicle. The directional skirt and angle skirt are respectively the upper and lower part of the spherical shell, the angle between the rotation axis $I$ of the directional skirt and the rotation axis $I I$ of the angle skirt is $\gamma$, in which the directional skirt can revolve around shaft $I$, angle skirt can revolve around shaft $I$. Through the directional skirt and angle skirt with the matching rotation can make docking skirt interface in the $0^{\circ} \sim 2 \gamma$ range within the rotation, as shown in Figure 2.

\footnotetext{
${ }^{a}$ Corresponding author : 350762275@qq.com
} 


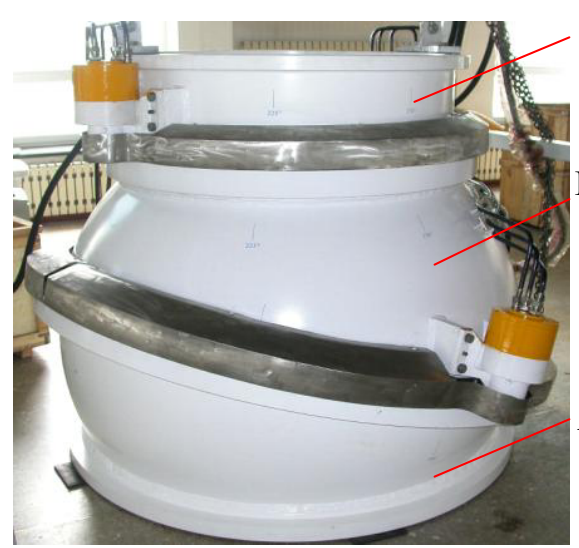

Figure 1. Composition of rotary docking skirt
Fixed skirt

Directional

Skirt

Angle skirt

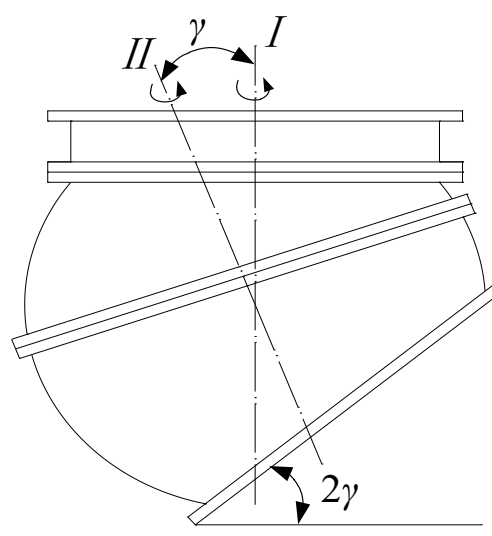

Figure 2. Rotation condition of the rotary docking skirt

\section{The establishment of the position matrix of the rotating docking skirt}

In order to facilitate deduction, this paper establishes a unified system. The midpoint of the contact plane of the fixed skirt and the directional skirt is $O_{1}, Y_{1}$ 's forward direction is forward direction of the lifeboat, $Z_{1}$ 's forward direction is vertical downward, $X_{1}$ 's forward direction is perpendicular to the plane of the Y1Z1 and points to the inside. In order to facilitate the subsequent calculation, here making the global coordinate system $O X Y Z$ and coordinate system $O_{1} X_{1} Y_{1} Z_{1}$ coincidence. The intersection of the axis $Z_{1}$ and the lower plane of the directional skirt is $O_{2}$, the intersection of the axis $I I$ and the lower plane of angle skirt is $O_{3}$, the intersection of the axis $I$ and the axis $I I$ is $O_{4}$, the intersection of the axis $I$ and the lower plane of the angle skirt is $O_{5}$, the center of docking skirt interface is $O_{6}$; We postulate the length of $O_{1} O_{2}$ is $D_{1}$ and $O_{2} O_{3}$ is $D_{2}$, and also postulate the length of $\mathrm{O}_{3} \mathrm{O}_{4}$ is $\mathrm{D}_{3}$ and $\mathrm{O}_{5} \mathrm{O}_{6}$ is $D_{4}$. The interface center of wrecked submarine is $O_{\mathrm{n}}$, as is shown in Figure 3.

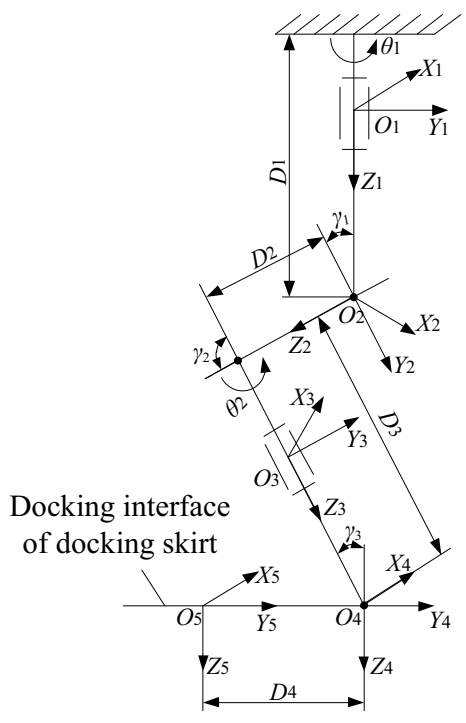

Docking interface of wrecked submarine

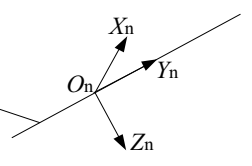

Figure 3. Rotary docking skirt docking motion coordinate system 
Here is the position transformation matrix of coordinate system $O_{1} X_{1} Y_{1} Z_{1}$ to $O_{6} X_{6} Y_{6} Z_{6}$ [6-8], the parameters are shown in Table 1.

$$
\begin{aligned}
{ }_{6}^{0} T= & \operatorname{Rot}\left(Z, \theta_{1}\right) \operatorname{Trans}\left(Z, D_{1}\right) \operatorname{Rot}\left(X,-\gamma_{1}\right) \operatorname{Trans}\left(Z, D_{2}\right) \operatorname{Rot}\left(X, \gamma_{2}\right) \times \\
& \operatorname{Trans}\left(Z, D_{3}\right) \operatorname{Rot}\left(Z, \theta_{2}\right) \operatorname{Trans}\left(Z, D_{4}\right) \operatorname{Rot}\left(X,-\gamma_{3}\right) \operatorname{Trans}\left(Y,-D_{5}\right)
\end{aligned}
$$

$$
{ }_{6}^{0} T={ }_{1}^{0} T_{2}^{1} T_{3}^{2} T_{4}^{3} T_{5}^{4} T_{6}^{5} T=\left[\begin{array}{cccc}
n_{x} & o_{x} & a_{x} & p_{x} \\
n_{y} & o_{y} & a_{y} & p_{y} \\
n_{z} & o_{z} & a_{z} & p_{z} \\
0 & 0 & 0 & 1
\end{array}\right]
$$

Table 1. Parameters in the coordinate system

\begin{tabular}{|c|c|c|c|}
\hline $\begin{array}{c}\text { serial number } \\
\text { of the link }\end{array}$ & $\gamma_{\mathbf{i}} /\left(^{\circ}\right)$ & $\boldsymbol{\theta}_{\mathbf{i}} /\left({ }^{\circ}\right)$ & $\boldsymbol{D}_{\mathbf{i}} / \mathbf{m m}$ \\
\hline 1 & 0 & $\theta_{1}$ & $D_{1}$ \\
\hline 2 & $-\gamma_{1}$ & 0 & $D_{2}$ \\
\hline 3 & $\gamma_{2}$ & 0 & $D_{3}$ \\
\hline 4 & $-\gamma_{3}$ & $\theta_{2}$ & $D_{4}$ \\
\hline 5 & 0 & 0 & $D_{5}$ \\
\hline
\end{tabular}

In equation:

$$
\begin{aligned}
& a_{x}=\left(-\cos \theta_{1} \sin \theta_{2}-\sin \theta_{1} \cos \gamma_{1} \cos \gamma_{2} \cos \theta_{2}-\sin \theta_{1} \sin \gamma_{1} \sin \gamma_{2} \cos \theta_{2}\right) \sin \gamma_{3} \\
&+\left(\sin \theta_{1} \cos \gamma_{1} \sin \gamma_{2}-\sin \theta_{1} \sin \gamma_{1} \cos \gamma_{2}\right) \cos \gamma_{3} \\
& a_{y}=\left(-\sin \theta_{1} \sin \theta_{2}+\cos \theta_{1} \cos \gamma_{1} \cos \gamma_{2} \cos \theta_{2}+\cos \theta_{1} \sin \gamma_{1} \sin \gamma_{2} \cos \theta_{2}\right) \sin \gamma_{3} \\
& \quad+\left(-\cos \theta_{1} \cos \gamma_{1} \sin \gamma_{2}+\cos \theta_{1} \sin \gamma_{1} \cos \gamma_{2}\right) \cos \gamma_{3} \\
& a_{z}=\left(-\sin \gamma_{1} \cos \gamma_{2} \cos \theta_{2}+\cos \gamma_{1} \sin \gamma_{2} \cos \theta_{2}\right) \sin \gamma_{3}+\left(\sin \gamma_{1} \sin \gamma_{2}+\cos \gamma_{1} \cos \gamma_{2}\right) \cos \gamma_{3} \\
& p_{x}=-\left[\left(-\cos \theta_{1} \sin \theta_{2}-\sin \theta_{1} \cos \gamma_{1} \cos \gamma_{2} \cos \theta_{2}-\sin \theta_{1} \sin \gamma_{1} \sin \gamma_{2} \cos \theta_{2}\right) \cos \gamma_{3}\right. \\
&\left.\left.-\sin \theta_{1} \cos \gamma_{1} \sin \gamma_{2} \sin \gamma_{3}+\sin \theta_{1} \sin \gamma_{1} \cos \gamma_{2} \sin \gamma_{3}\right)\right] D_{5}+\left(\sin \theta_{1} \cos \gamma_{1} \sin \gamma_{2}-\sin \theta_{1} \sin \gamma_{1} \cos \gamma_{2}\right) D_{4} \\
&+\left(\sin \theta_{1} \cos \gamma_{1} \sin \gamma_{2}-\sin \theta_{1} \sin \gamma_{1} \cos \gamma_{2}\right) D_{3}-\sin \theta_{1} \sin \gamma_{1} D_{2} \\
& p_{y}=-\left[\left(-\sin \theta_{1} \sin \theta_{2}+\cos \theta_{1} \cos \gamma_{1} \cos \gamma_{2} \cos \theta_{2}+\cos \theta_{1} \sin \gamma_{1} \sin \gamma_{2} \cos \theta_{2}\right) \cos \gamma_{3}\right. \\
&\left.-\left(-\cos \theta_{1} \cos \gamma_{1} \sin \gamma_{2}+\cos \theta_{1} \sin \gamma_{1} \cos \gamma_{2}\right) \sin \gamma_{3}\right] D_{5}+\left(-\cos \theta_{1} \cos \gamma_{1} \sin \gamma_{2}+\cos \theta_{1} \sin \gamma_{1} \cos \gamma_{2}\right) D_{4} \\
&+\left(-\cos \theta_{1} \cos \gamma_{1} \sin \gamma_{2}+\cos \theta_{1} \sin \gamma_{1} \cos \gamma_{2}\right) D_{3}+\cos \theta_{1} \sin \gamma_{1} D_{2} \\
& p_{z}=-\left[\left(-\sin \gamma_{1} \cos \gamma_{2} \cos \theta_{2} \cos \gamma_{3}+\cos \gamma_{1} \sin \gamma_{2} \cos \theta_{2} \cos \gamma_{3}\right)-\left(\sin \gamma_{1} \sin \gamma_{2} \sin \gamma_{3}+\cos \gamma_{1} \cos \gamma_{2} \sin \gamma_{3}\right)\right] D_{5} \\
&+\left(\sin \gamma_{1} \sin \gamma_{2}+\cos \gamma_{1} \cos \gamma_{2}\right) D_{4}+\left(\sin \gamma_{1} \sin \gamma_{2}+\cos \gamma_{1} \cos \gamma_{2}\right) D_{3}+\cos \gamma_{1} D_{2}+D_{1}
\end{aligned}
$$

\section{Analysis on the Movement of Rotary Docking Skirt}

In this article, $\gamma_{2}=90^{\circ}$. so $a_{\mathrm{x}}, a_{\mathrm{y}}$ and $a_{\mathrm{z}}$ can be simplified as:

$$
a_{x}=-\cos \theta_{1} \sin \theta_{2} \sin \gamma_{3}-\sin \theta_{1} \sin \gamma_{1} \cos \theta_{2} \sin \gamma_{3}+\sin \theta_{1} \cos \gamma_{1} \cos \gamma_{3}
$$




$$
\begin{gathered}
a_{y}=-\sin \theta_{1} \sin \theta_{2} \sin \gamma_{3}+\cos \theta_{1} \sin \gamma_{1} \cos \theta_{2} \sin \gamma_{3}-\cos \theta_{1} \cos \gamma_{1} \cos \gamma_{3} \\
a_{z}=\cos \theta_{2} \cos \gamma_{1} \sin \gamma_{3}+\sin \gamma_{1} \cos \gamma_{3}
\end{gathered}
$$

We postulate the docking interface center of wrecked submarine is coordinate system $O_{\mathrm{n}} X_{\mathrm{n}} Y_{\mathrm{n}} Z_{\mathrm{n}}$, the position matrix $N$ of the wrecked submarine's docking interface center can be regarded as $O_{1} X_{1} Y_{1} Z_{1}$ which is respectively moving $X_{\mathrm{n}}$ along the axis $X_{1}$, moving $Y_{\mathrm{n}}$ along the axis $Y_{1}$ and moving $Z_{\mathrm{n}}$ along the axis $Z_{1}$, rotate $\beta_{2}$ around the axis $Y_{1}$, rotate the $\beta_{1}$ around the axis $X_{1}$.

$$
\begin{aligned}
N & =\operatorname{Trans}\left(X, X_{n}\right) \operatorname{Trans}\left(Y, Y_{n}\right) \operatorname{Trans}\left(Z, Z_{n}\right) \operatorname{Rot}\left(Y, \beta_{2}\right) \operatorname{Rot}\left(X, \beta_{1}\right) \\
& =\left[\begin{array}{cccc}
\cos \beta_{2} & \sin \beta_{1} \sin \beta_{2} & \sin \beta_{2} \cos \beta_{1} & X_{n} \\
0 & \cos \beta_{1} & -\sin \beta_{1} & Y_{n} \\
-\sin \beta_{2} & \sin \beta_{1} \cos \beta_{2} & \cos \beta_{1} \cos \beta_{2} & Z_{n} \\
0 & 0 & 0 & 1
\end{array}\right]
\end{aligned}
$$

By the above formula, the direction cosine of the axis $Z$ unit principal vector of the coordinate system $O_{\mathrm{n}} X_{\mathrm{n}} Y_{\mathrm{n}} Z_{\mathrm{n}}$ relative to the coordinate system $O_{1} X_{1} Y_{1} Z_{1}$ is:

$$
Z_{N}=\left[\begin{array}{lll}
\sin \beta_{2} \cos \beta_{1} & -\sin \beta_{2} & \cos \beta_{1} \cos \beta_{2}
\end{array}\right]
$$

The direction cosine of the axis $\mathrm{Z}$ unit principal vector of the coordinate system $O_{6} X_{6} Y_{6} Z_{6}$ relative to the coordinate system $O_{1} X_{1} Y_{1} Z_{1}$ is:

$$
{ }_{6}^{0} a=\left[\begin{array}{lll}
a_{x} & a_{y} & a_{z}
\end{array}\right]
$$

In order to ensure docking skirt interface and wrecked submarine interface parallel docking , should make:

$$
{ }_{6}^{0} a=Z_{N}
$$

We can obtain directional skirt angle $\theta_{1}$ and angle skirt angle $\theta_{2}$ from equation (15):

$$
\begin{gathered}
\cos \theta_{1}=\frac{\sin \beta_{1} \cos \gamma_{1} \cos \gamma_{3}-\sin \beta_{1} \cos \theta_{2} \sin \gamma_{1} \sin \gamma_{3}-\sin \beta_{2} \cos \beta_{1} \sin \theta_{2} \sin \gamma_{3}}{\left(\sin \theta_{2} \sin \gamma_{3}\right)^{2}+\left(\cos \theta_{2} \sin \gamma_{1} \sin \gamma_{3}-\cos \gamma_{1} \cos \gamma_{3}\right)^{2}} \\
\cos \theta_{2}=\frac{\cos \beta_{1} \cos \beta_{2}-\sin \gamma_{1} \cos \gamma_{3}}{\cos \gamma_{1} \sin \gamma_{3}}
\end{gathered}
$$

In order to ensure the docking skirt interface and the wrecked submarine interface coincidence, the life-saving vehicle needs to travel distance is:

$$
L_{x}=X_{n}-P_{x}, \quad L_{y}=Y_{n}-P_{y}, \quad L_{z}=Z_{n}-P_{z}
$$

The optimal distance is obtained, as shown in Figure 4:

$$
L_{\min }=\sqrt{\left(X_{n}-P_{x}\right)^{2}+\left(Y_{n}-P_{y}\right)^{2}+\left(Z_{n}-P_{z}\right)^{2}}
$$




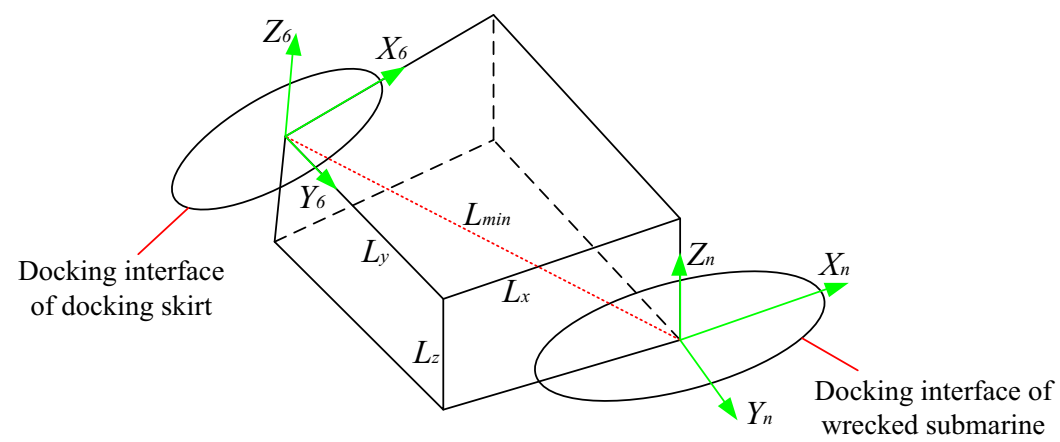

Figure 4. The best route for a lifeboat

\section{Determination of the range of rotary docking operations}

Because of the position of the wrecked submarine is random in the seabed. Therefore, it is need to get the rotation angle of the directional skirt and angle skirt when the wrecked submarine is in different heel angle and trim angle. It is assumed that the range of the heel angle and trim angle of the wrecked submarine is all $0^{\circ} \sim 60^{\circ}$, and the samples were sampled at intervals of $10^{\circ}$. In this article, $\gamma_{1}=60^{\circ}$, $\gamma_{2}=90^{\circ}$ and $\gamma_{3}=30^{\circ}$. Based on the range of angle cosine of the directional skirt and angle skirt in the kinematics model above, using Matlab to program can get the angle of directional skirt and angle skirt , as shown in Figure 5 and Figure 6.

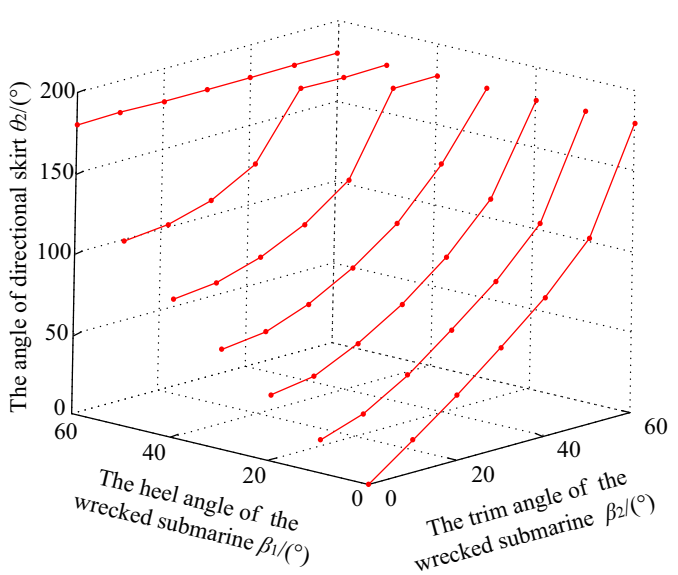

Figure 5. The angle of rotation by angle skirt

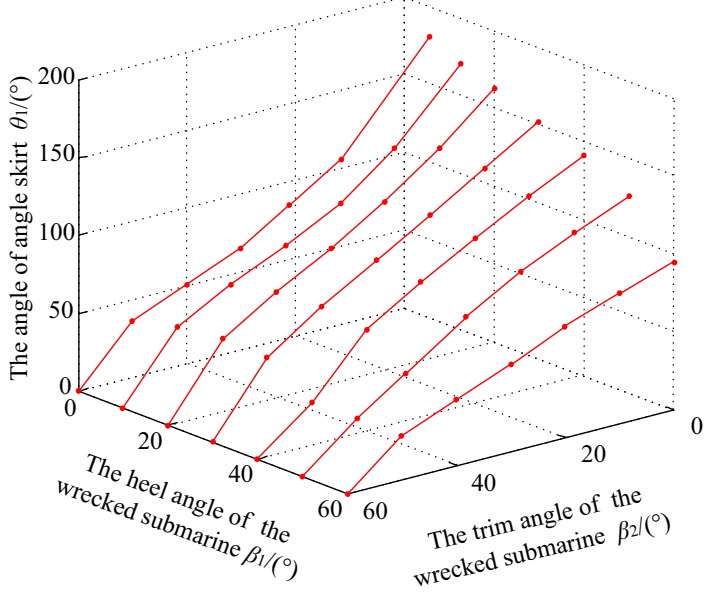

Figure 6. The angle of rotation by directional skirt

\section{Conclusion}

Through the study of the double spherical shell rotating docking skirt. And the fixed skirt, the directional skirt, the angle skirt are simplified as the connecting rod. We get the pose equation and the kinematic model by analyzing the working principle of the rotating docking skirt. The angle of rotation of the directional skirt and the angle skirt is obtained when the wrecked submarine is in different trim and heel angles. Through the directional skirt and angle skirt with the matching rotation can make docking skirt interface in the $0^{\circ} \sim 2 \gamma$ range within the rotation, to complete the docking skirt and the wrecked submarine docking. The MATLAB software is used to visualize the rotation angle of 
fixed skirt and directional skirt, and the correctness of the theoretical analysis of the rotary skirt docking is proved, which lays a good foundation for the development of the control of the double spherical shell rotating docking skirt in future.

\section{References}

1. L. Q. WANG, D. D. TANG, J. R. Wu, Z. L. Zhang, Research on Structure and Kinematics of a Novel Mating Skirt on the Underwater Vehicle [J]. China Mechanical Engineering, 19, 2814$2818(2008)$

2. X. Y. Meng, W. Tian. Comments on the nuclear submarine escape and rescue system development of foreign countries [J]. Ship Science and Technology, 34, 137-143 (2012)

3. B. G. Fu, Q. X. Meng, H. M. Liu, Current situation and development trend of deep submergence rescue vehicles [J]. The Ocean Engineering, 25, 120-126 (2007)

4. Owen F. Submarine rescue with an Australian twang, indepth rescue [J]. A Newsletter for Submarine Rescue Professionals, 1, 1-6 (2004)

5. D. D. TANG, L. Q. WANG, Q. X. Meng, J. R. Wu, Z. L. Zhang, Structure design and carrying capacity analysis of mating skirt on the DSRV [J], 31, 1-4 (2009)

6. X. Zhang, Z. L. Zheng, Y. Qi, Parameter Identification and Calibration of D-H Model for 6-DOF Serial Robots [J]. Robot, 38, 360-370 (2016)

7. Y. Gan, J. L. Wang, F. J. Sun, Optimal Design of D-H Parameters of a 6R Robot for a Prescribed Workspace [J]. China Mechanical Engineering, 25, 3003-3007+3011 (2014)

8. F. Y. Guo, T. Mei, J. H. Zhao, Problems and Improvement of D-H Method for Establishing Connecting Rod Coordinate System [J]. China Mechanical Engineering, 25, 2710-2714 (2014) 